\title{
First report of leaf blight caused by Fusarium proliferatum on Polygonatum cyrtonema in China
}

\author{
Xianzhi Zhou ${ }^{1,2}$ (1) $\cdot{\text { Baorong } \text { Rao }^{3} \cdot \text { Yonghe Chen }^{3} \cdot \text { Chengfeng Cai }}^{1}$ \\ Received: 12 May 2020 / Accepted: 12 November 2020 / Published online: 25 November 2020 \\ (C) Società Italiana di Patologia Vegetale (S.I.Pa.V.) 2020
}

Keywords Fusarium proliferatum $\cdot$ Leaf blight $\cdot$ Pathogenicity test $\cdot$ Polygonatum cyrtonema

Polygonatum cyrtonema Hua is an important perennial medicinal herb plant. From March 2015 to September 2019, leaf blight symptoms were observed on P. cyrtonema plants in the planting regions in Chongren, Kekeng and Gulin Villages, Guangze County, China, with disease incidence of approximately $20-35 \%$ in the above district. The colonies were white to light pink with woolly purple aerial mycelium on potato dextrose agar (PDA), the plate reverses of the colonies were purple. Microscopic observations showed hyaline and septate hyphae, slightly curved macroconidia with three to four septa measuring 18.5 to $36.5 \times 2.5$ to $4.0 \mu \mathrm{m}(n=20)$, and microconidia measuring 7.8 to $11.5 \times 2.5$ to $3.5 \mu \mathrm{m}(n=50)$. These morphological characteristics were consistent with the description of Fusarium genus (Leslie and Summerell 2006). To confirm the identity of isolates (DHHJYK1 and DHHJYK2), the rDNA internal transcribed spacer region (ITS) and translation elongation factor 1 alpha gene (EF-1a) gene were amplified using primers ITS1 / ITS4 (White et al. 1990) and EF-1 / EF-2 (O’Donnell et al. 1998), respectively. The resulting sequences for ITS and EF-1a were submitted to GenBank with accession numbers (MN461564, MN461565,

Xianzhi Zhou and Baorong Rao contributed equally to this work.

Xianzhi Zhou

xianzhizhou@126.com

1 Agricultural Bio-resources Research Institute, Fujian Academy of Agricultural Sciences, Wusi Road 247, Fuzhou 350003, People's Republic of China

2 Scientific Observation and Experiment Station of Fuzhou, Ministry of Agriculture and Rural Affairs, 350003 Fuzhou, People's Republic of China

3 Nanping Institute of Agricultural Scieence in Fujian Province, Nanping 354200, People's Republic of China
MT265244 and MT265245). The ITS sequences of the two isolates showed $100.0 \%$ similarity with ITS sequence of $F$. proliferatum in GenBank (KU891521.1). The EF-1a sequences of the two isolates showed $99.7 \%$ similarity with EF-1a sequence of $F$. proliferatum in GenBank (AF160280). Leaves from 3-year-old healthy plants of $P$. cyrtonema were wounded by a sterilised needle and inoculated by $5 \mathrm{~mm}$ mycelial plugs of the two isolates grown for 5 days on PDA. Control leaves were inoculated by sterile PDA plugs. There were six leaves for each replicates. The leaves were incubated at $12 \mathrm{~h}$ light/dark, 20 $25^{\circ} \mathrm{C}$, and $80 \%$ relative humidity. Blight symptoms were observed on all inoculated leaves after four days, but no symptoms were seen oncontrol leaves. Koch's postulates were fulfilled by reisolation of $F$. proliferatum from diseased leaves. This is first report of $F$. proliferatum as a pathogen of $P$. cyrtonema in North Fujian, China.

Acknowledgments The research was financed by the Science and Technology Innovation Team Construction Project of FAAS (No. STIT2017-2-8), Central Public-interest Scientific Institution Basal Research Fund for Chinese Academy of Tropical Agricultural Sciences (No. 1630052017020-3).

\section{References}

Leslie JF, Summerell BA (2006) The Fusarium Laboratory Manual. Blackwell Publishing, Oxford

O’Donnell K, Kistler HC, Cigelnik E, Ploetz RC (1998) Multiple evolutionary origins of the fungus causing Panama disease of banana: Concordant evidence from nuclear and mitochondrial gene genealogies. Proc Natl Acad Sci USA 95:2044-2049

White TJ, Bruns T, Lee S, Taylor J (1990) Amplification and direct sequencing of fungal ribosomal RNA genes for phylogenetics. In: Innis MA, Gelfand DH, Sninsky JJ, White TJ (eds) PCR protocols: a guide to methods and applications. Academic, San Diego, pp 315322 